\title{
644 TUMOR-MEDIATED SUPPRESSIVE SIGNALING AND HYPOXIA SUPPORTS ALTERED CHROMATIN LANDSCAPES THAT LIMIT THE TRANSCRIPTIONAL AND FUNCTIONAL POTENTIAL OF TERMINAL T CELL EXHAUSTION
}

Rhodes Ford*, Natalie Rittenhouse, Nicole Scharping, Paolo Vignali, Greg Delgoffe, Amanda Poholek. University of Pittsburgh, Pittsburgh, PA, United States

Background CD8 $+\mathrm{T}$ cells are a fundamental component of the anti-tumor response; however, tumor-infiltrating CD8 $+\mathrm{T}$ cells (TIL) are rendered dysfunctional by the tumor microenvironment. CD8 + TIL display an exhausted phenotype with decreased cytokine expression and increased expression of coinhibitory receptors (IRs), such as PD-1 and Tim-3. The acquisition of IRs mark the progression of dysfunctional TIL from progenitors (PD-1Low) to terminally exhausted (PD-1 + Tim-3+). How the chromatin landscape changes during this progression has not been described.

Methods Using a low-input ChIP-based assay called Cleavage Under Targets and Release Using Nuclease (CUT\&RUN), we have profiled the histone modifications at the chromatin of tumor-infiltrating CD8 $+\mathrm{T}$ cell subsets to better understand the relationship between the epigenome and the transcriptome as TIL progress towards terminal exhaustion.

Results We have identified two epigenetic characteristics unique to terminally exhausted cells. First, we have identified a unique set of genes, characterized by active histone modifications that do not have correlated gene expression. These regions are enriched for AP-1 transcription factor motifs, yet most AP-1 family factors are actively downregulated in terminally exhausted cells, suggesting signals that promote downregulation of AP-1 expression negatively impacts gene expression. We have shown that inducing expression of AP-1 factors with a 41BB agonist correlates with increased expression of these anticorrelated genes. We have also found a substantial increase in the number of genes that exhibit bivalent chromatin marks, defined by the presence of both active (H3K4me3) and repressive (H3K27me3) chromatin modifications that inhibit gene expression. These bivalent genes in terminally exhausted $\mathrm{T}$ cells are not associated with plasticity and represent aberrant hypermethylation in response to tumor hypoxia, which is necessary and sufficient to promote downregulation of bivalent genes.

Conclusions Our study defines for the first time the roles of costimulation and the tumor microenvironment in driving epigenetic features of terminally exhausted tumor-infiltrating $\mathrm{T}$ cells. These results suggest that terminally exhausted $\mathrm{T}$ cells have genes that are primed for expression, given the right signals and are the basis for future work that will elucidate that factors that drive progression towards terminal $\mathrm{T}$ cell exhaustion at the epigenetic level and identify novel therapeutic targets to restore effector function of tumor $\mathrm{T}$ cells and mediate tumor clearance.

http://dx.doi.org/10.1136/jitc-2021-SITC2021.644 